\title{
Real-space condensation in a dilute Bose gas at low temperature
}

I. O. Kulik

Citation: Low Temperature Physics 27, 873 (2001); doi: 10.1063/1.1414580

View online: $\mathrm{http}: / / d x . d o i . o r g / 10.1063 / 1.1414580$

View Table of Contents: http://aip.scitation.org/toc/ltp/27/9

Published by the American Institute of Physics

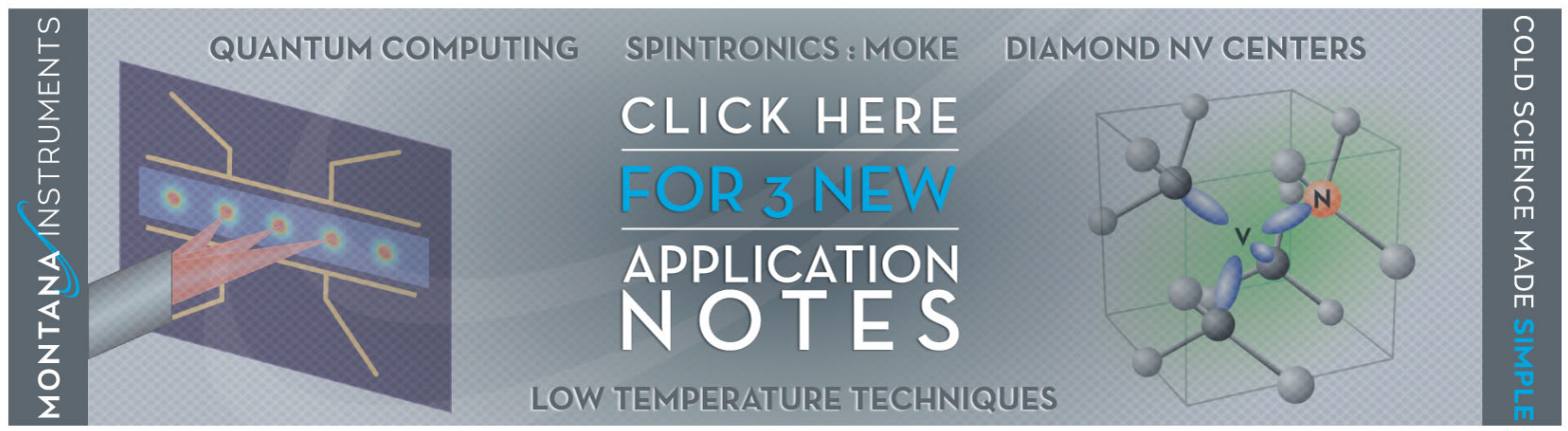




\title{
Real-space condensation in a dilute Bose gas at low temperature
}

\author{
I. O. Kulik*
}

Department of Physics, Bilkent University, Bilkent, Ankara 06533, Turkey

(Submitted May 16, 2001)

Fiz. Nizk. Temp. 27, 1179-1182 (September-October 2001)

We show with a direct numerical analysis that a dilute Bose gas in an external potential-which is chosen for simplicity as a radial parabolic well—undergoes at a certain temperature $T_{c}$ a phase transition to a state supporting a macroscopic fraction of particles at the origin of the phase space $(\mathbf{r}=0, \mathbf{p}=0)$. Quantization of particle motion in a well wipes out the sharp transition but supports a distribution of a radial particle density $\rho(r)$ peaked at $r=0$ (a real-space condensate) as well as a phase-space Wigner distribution density $W(\mathbf{r}, \mathbf{p})$ peaked at $\mathbf{r}=0$ and $\mathbf{p}=0$ below a crossover temperature $T_{c}^{*}$ of order of $T_{c}$. A fixed-particle-number canonical ensemble, which is a combination of the fixed- $N$ condensate part and the fixed- $\mu$ excitation part, is suggested to resolve the difficulty of large fluctuation of the particle number $(\delta N \sim N)$ in the Bose-Einstein condensation problem treated within the orthodox grand canonical ensemble formalism. (C) 2001 American Institute of Physics. [DOI: 10.1063/1.1414580]

The phenomenon of Bose-Einstein (BE) condensation (see textbooks, e.g., Refs. 1-3) manifests itself in the formation of macroscopic fraction of zero-momentum particles uniformly distributed in a coordinate space. Such transition was recently observed in laser-trapped, evaporation-cooled atomic vapors $^{4-6}$ in magnetic traps (see recent reviews ${ }^{7-9}$ ). We will show by a direct numerical analysis, partly similar to and sometimes overlapping with the previous theoretical works on the subject, ${ }^{10-13}$ that a Bose gas in an external confining potential condenses at low temperature to a position of minimum potential energy; the particles of that "condensate" also have zero kinetic energy. Quantization of particle states in a well makes the real-space condensation a continuous transition rather than a phase transition but still supports a macroscopic fraction of particles near the origin of the coordinate space below a crossover temperature $T_{c}^{*}$ which is of the order of Bose-condensation temperature $T_{c}$.

Experimental realization of $\mathrm{BE}$ condensation implies confinement of a dilute gas within some region of space in a "trap" cooled by its interaction with an "optical molasses" created by laser irradiation ${ }^{14}$ and finally cooled to microwave-range temperature by evaporative cooling. ${ }^{11}$ Bose gas in a trap may be considered to be interacting with two thermal reservoirs, the first one representing the thermal environment (walls, blackbody radiation at temperature $T_{1}$ ) and the second one the optical molasses at temperature $T_{2}$ $\ll T_{1}$. The equilibrium distribution of particles $f(\mathbf{p}, \mathbf{r}, t)$ can be obtained by solving the Boltzmann kinetic equation

$$
\frac{d f}{d t}=\hat{I}_{1}\{f\}+\hat{I}_{2}\{f\}
$$

where $\hat{I}_{1}$ is the interaction term (Stoss integral) corresponding to coupling with a media 1 , and $\hat{I}_{2}$, respectively, with media 2 . If we choose for simplicity the relaxation time approximation for $\hat{I}_{1,2}$,

$$
\hat{I}_{i}=-\frac{f-f_{i}}{\tau_{i}}
$$

then the solution for the equilibrium state will be

$$
f=\frac{\tau_{1}^{-1} f_{1}^{0}+\tau_{2}^{-1} f_{2}^{0}}{\tau_{1}^{-1}+\tau_{2}^{-1}}
$$

The relaxation rate $\tau_{2}^{-1}$ is proportional to the laser intensity $P$. At large intensity, assuming $\tau_{2}^{-1} \gg \tau_{1}^{-1}$, Eq. (3) gives $f$ $\simeq f_{2}^{0}$.

In a semiclassical approximation, the particle energy is

$$
\varepsilon=\frac{\mathbf{p}^{2}}{2 m}+\frac{1}{2} m \Omega^{2} \mathbf{r}^{2},
$$

where the thermodynamic potential $\Omega=-T \ln Z, Z$ is the grand partition function (assuming zero spin of particles)

$$
Z=\int \frac{d \mathbf{p} d \mathbf{r}}{(2 \pi \hbar)^{3}} \ln \left(1-e^{(\mu-\varepsilon) / T}\right),
$$

where $\hbar$ is Planck's constant. The chemical potential $\mu$ is determined from (5) to satisfy an equation

$$
N=\int \frac{d \mathbf{p} d \mathbf{r}}{(2 \pi \hbar)^{3}} \frac{1}{e^{(\varepsilon-\mu) / T}-1},
$$

where $N$ is the number of particles. After integration over the directions of $\mathbf{r}$ and $\mathbf{p}$ we obtain

$$
\begin{aligned}
N= & \frac{(4 \pi)^{2}}{(2 \pi \hbar)^{3}}(2 m T)^{3 / 2}\left(\frac{2 T}{m \Omega^{2}}\right)^{3 / 2} \\
& \times \int_{0}^{\infty} x^{2} d x \int_{0}^{\infty} y^{2} d y \frac{1}{e^{x^{2}+y^{2}-\zeta}-1},
\end{aligned}
$$

where $\zeta<0$ is the chemical potential in appropriate dimensionless units.

At low temperature, no nonzero value of $\zeta$ can satisfy Eq. (7). It therefore vanishes at a temperature $T=T_{c 0}$ determined from the condition $\zeta=0$, thus giving

$$
T_{c 0}=\hbar \Omega(N / \zeta(3))^{1 / 3}=0.94 \hbar \Omega N^{1 / 3},
$$




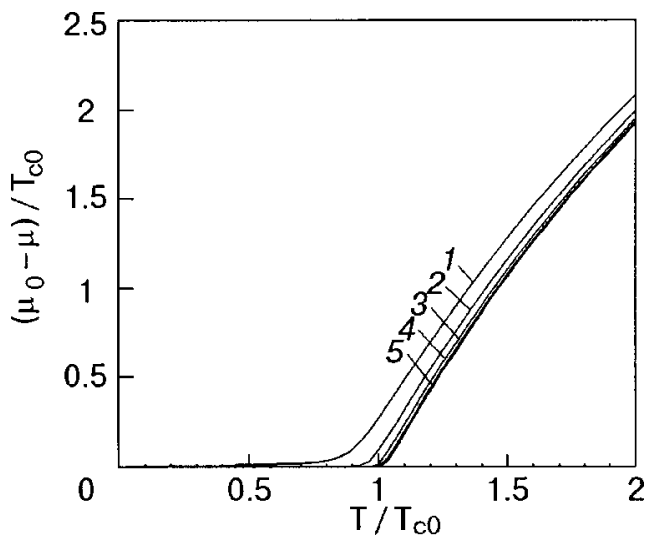

FIG. 1. Chemical potential versus temperature for various values of $N: 10^{2}$ $(1), 10^{3}(2), 10^{4}(3), 10^{5}(4), 10^{6}(5)$.

where $\zeta(z)$ is the Riemann zeta function. Below $T_{c 0}$, $\zeta$ remains equal to zero with the total number of particles $N_{0}$ having both $\mathbf{r}=0$ and $\mathbf{p}=0$ values, determined from

$$
N_{0}=\left(1-\frac{T^{3}}{T_{c 0}^{3}}\right) N \text {. }
$$

Of course, the $\mathbf{r}=0, \mathbf{p}=0$ state is not allowed quantummechanically, and the derivation leading to Eqs. (6), (7) needs modification. The energy of a particle in a parabolic well, Eq. (4), is

$$
\varepsilon=\hbar \Omega\left(n_{1}+n_{2}+n_{3}+3 / 2\right), \quad n_{i}=0,1 \ldots .
$$

Then the normalization condition, Eq. (6), reduces to

$$
N=\sum_{n=0}^{\infty} \frac{S_{n}}{\eta e^{n x}-1}
$$

with

$$
S_{n}=\sum_{n_{1}, n_{2}, n_{3}=0}^{n} \delta_{n_{1}+n_{2}+n_{3}, n}=\frac{1}{2}(n+1)(n+2)
$$

and $\eta=\exp \left(\left(\mu_{0}-\mu\right) / T\right), x=\hbar \Omega / T ; \mu_{0}$ is the value of the chemical potential at $T=0\left(\mu_{0}=3 / 2 \hbar \Omega\right)$.

The solution of Eq. (10) shows the dependence $\mu(T)$ (Fig. 1) with a crossover between almost linear dependence above the crossover temperature $T_{c}^{*}$ and a practically zero value below that temperature. The value of $T_{c}^{*}$ is very near to $T_{c 0}$ at large number of particles, $N \gg 1$.

The particle density distribution is expressed through the sum of Hermite polynomials. ${ }^{15}$ Employing the identity for these polynomials

$$
\begin{aligned}
& \sum_{n_{1}+n_{2}+\ldots+n_{r}=n} \prod_{k=1}^{r} \frac{H_{n_{k}}^{2}\left(x_{k}\right)}{2^{n_{k}} n_{k} !} \\
& =\sum_{m=0}^{n} r_{n-m} \frac{1}{2^{m} m !} H_{m}^{2}\left(\left(\sum_{k=1}^{r} x_{k}^{2}\right)^{1 / 2}\right),
\end{aligned}
$$

where $r_{m}=1$ for $m$ even and $r_{m}=0$ for $m$ odd, we receive by putting $r=3$

$$
n(\mathbf{r})=\frac{e^{-r^{2}}}{\pi^{3 / 2}} \sum_{m=0}^{\infty} \frac{H_{m}^{2}(r)}{2^{m} m !} \sum_{k=0}^{\infty} \frac{1}{\eta e^{(m+2 k) x}-1} .
$$

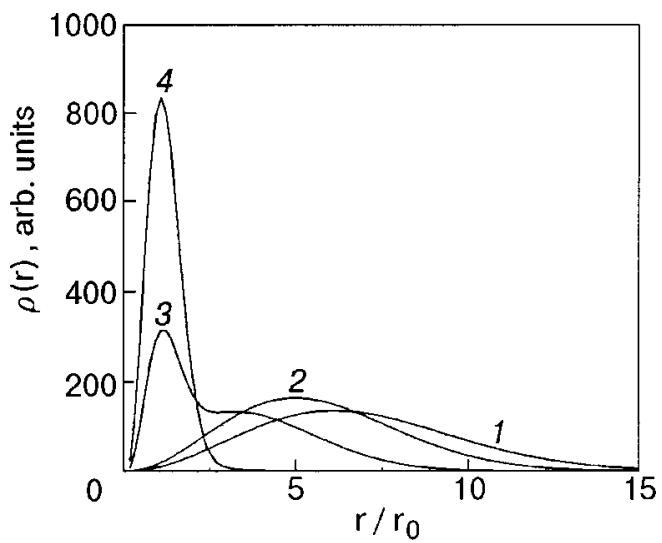

FIG. 2. Radial density distribution $\rho(r)=4 \pi r^{2} n(r)$ for $N=1000$ and various temperatures: $T / T_{c 0}=0.2$ (1), 0.8 (2), 1.4 (3), 2.0 (4).

Figure 2 shows the radial density distribution $\rho(r)$ $=4 \pi r^{2} n(r)$ at various temperatures. Below $T_{c}^{*}, \rho(r)$ displays a second maximum at small $r$, which grows in amplitude as the temperature decreases: the real-space condensate. The formation of such a condensate is even more explicit in the evolution of the $z$-projected density distribution, Fig. 3, as the temperature is decreased from above to below $T_{c 0}$.

At zero temperature, all excited particles above the condensate vanish. The joint momentum-coordinate distribution function (the Wigner distribution function ${ }^{16}$ ) takes a value

$$
W(\mathbf{p}, \mathbf{r})=\frac{N_{0}}{\pi r_{0}} e^{-p^{2} r_{0}^{2}} e^{-r^{2} / r_{0}^{2}},
$$

where $r_{0}=(\hbar / m \Omega)^{1 / 2}$ is the zero-point oscillation amplitude in a parabolic well.

The question remains, how to reconcile the above results with the free-space Bose-Einstein condensation. The BE condensation temperature equals ${ }^{1}$

$$
T_{0}=3.31 \frac{\hbar^{2}}{m} n^{2 / 3} \text {. }
$$

The average density of particles in a well above the condensation temperature is

$$
\bar{n} \sim N / T^{3}, \text { where } \bar{r}=\left(\frac{T}{m \Omega}\right)^{1 / 2} \sim r_{0} N^{1 / 6}\left(T / T_{0}\right)^{1 / 2},
$$

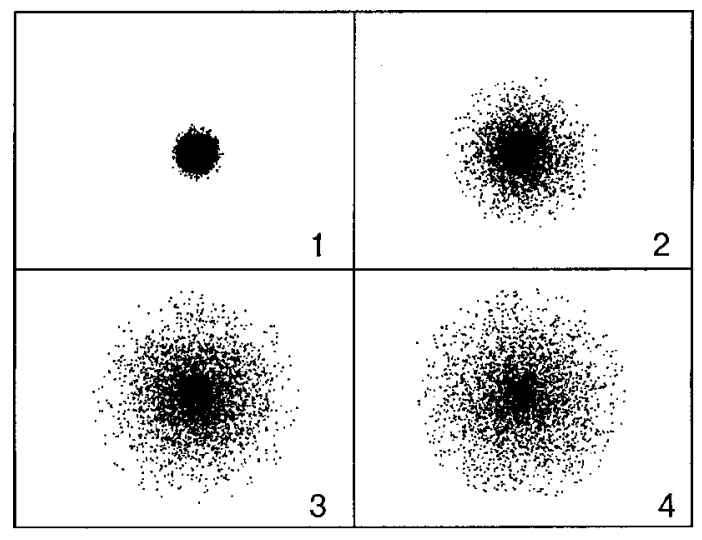

FIG. 3. Side view of particle distribution: $1-T=0.2 T_{c 0}, 2-T=0.8 T_{c 0}$, $3-T=1.4 T_{c 0}, 4-T=2.0 T_{c 0}$. 
where $\bar{r}$ is a confinement radius (mean radius of the gaseous cloud). It is related to the minimal quantum radius $r_{0}$ as $\bar{r}$ $\sim r_{0} N^{1 / 6}\left(T / T_{c}\right)^{1 / 2}$. By putting $T \sim T_{c}^{*}$ as defined above, we obtain $T$ of the order of the BE condensation temperature (14). Therefore, the phenomenon we discussed is just the BE condensation mechanism, ${ }^{1}$ except that in a trap the condensation occurs in both the momentum and coordinate spaces or, if we choose to explore the behavior of a dilute lowtemperature Bose gas in real space, it will condense there, making up a high-density globular fraction coexisting with the spatially dispersed "excitations" in a region of size comparable to the thermal confinement radius $\bar{r}$.

In the grand canonical ensemble which we so far have been considering, the number of particles is not fixed. The mean square fluctuation of particle number in a state $\alpha$ is $\left\langle\delta n_{\alpha}^{2}\right\rangle=n_{\alpha}\left(n_{\alpha}+1\right)$. In a condensate, by putting $\left\langle n_{\alpha=0}\right\rangle$ $=N_{0}$ we get $\mu \simeq \varepsilon_{0}-T / N_{0}$ and $\left\langle\delta n_{0}^{2}\right\rangle^{1 / 2} \simeq N_{0}$. This means a huge fluctuation of particle number $\delta N \sim N$ at $T \ll T_{0}$, an unrealistic property of the model. ${ }^{17}$

In a canonical ensemble, which better fits to experiments with dilute gases in traps, the average value of the condensate population is given by

$\left\langle n_{0}\right\rangle=\frac{\sum_{n_{0}=0}^{N} n_{0} \sum_{\left\{n_{\alpha}\right\}^{\prime}} e^{-\beta \Sigma_{\alpha>0}\left(\varepsilon_{\alpha}-\varepsilon_{0}\right) n_{\alpha}} \delta_{\Sigma_{\alpha>0} n_{\alpha}, N-n_{0}}}{\sum_{n_{0}=0}^{N} \sum_{\left\{n_{\alpha}\right\}^{\prime}} e^{-\beta \Sigma_{\alpha>0}\left(\varepsilon_{\alpha}-\varepsilon_{0}\right) n_{\alpha}} \delta_{\Sigma_{\alpha>0} n_{\alpha}, N-n_{0}}}$,

where $\left\{n_{\alpha}\right\}^{\prime}$ stands for a collection of all state numbers except $n_{0}$, and $\beta=1 / T$. The average over such states does not fluctuate strongly and therefore can be replaced by its grand canonical value corresponding to an appropriate choice of chemical potential $\mu=\mu_{N-n_{0}}$. We thus get

$$
\left\langle n_{0}\right\rangle \cong \frac{\sum_{n_{0}=0}^{N} n_{0} Z_{N-n_{0}}}{\sum_{n_{0}=0}^{N} Z_{N-n_{0}}},
$$

where $Z_{n}=\mathrm{e}^{-\beta \Omega_{n}}$, and $\Omega_{n}$ is the thermodynamic potential of the grand canonical ensemble. ${ }^{1}$

The quantity $Z_{n}=\mathrm{e}^{-\beta N}$ is not exponentially small for a number of particles $n$ smaller than the Bose-condensate fraction, $n<N_{0}$. Therefore, we can change expression (17) to

$$
\left\langle n_{0}\right\rangle \cong \frac{\sum_{n_{0}=N_{0}}^{N} n_{0} \mathrm{e}^{-\beta \Omega_{N-n_{0}}}}{\sum_{n_{0}=N_{0}}^{N} \mathrm{e}^{-\beta \Omega_{N-n_{0}}}} .
$$

The quantity $\Omega_{n}$ is strongly peaked at $n=N_{0}$, thus giving $\left\langle n_{0}\right\rangle \simeq N_{0}$ and, similarly, $\left\langle\delta n_{0}^{2}\right\rangle^{1 / 2} \sim \sqrt{N_{0}}$ rather than $\left\langle\delta n_{0}^{2}\right\rangle^{1 / 2} \sim N_{0}$ as in the orthodox grand canonical ensemble. Indeed, at $N \ll N_{0}$ (corresponding to $T \gg T_{0}$ ) we obtain for the thermodynamic potential $\Omega_{N}$ a value $\Omega_{N} \simeq-N T$, and $Z_{N}$ $\simeq \mathrm{e}^{N}$. This agrees with the conclusion, reached in a different way in Ref. 12, that the thermodynamic properties of a Bose condensate in a trap with fixed total number of particles are very similar to those in the orthodox grand canonical ensemble with a fixed average number of particles. The above results are consistent with a known statement that the BoseEinstein condensation temperature $T_{0}$ is the same in the canonical and in the grand canonical ensembles. ${ }^{2}$

In conclusion, I hope I have met the goal of elucidating in a direct way the properties of the low-temperature state of an ideal Bose gas of finite-size, finite-particle-number systems. I express my deep gratitude to Prof. B. Tanatar for stimulating discussions and help.

*Also at: B. Verkin Institute for Low Temperature Physics and Engineering of the National Academy of Sciences of Ukraine, 47 Lenin. Ave., Kharkov 61103, Ukraine.

E-mail: kulik@fen.bilkent.edu.tr

${ }^{1}$ L. D. Landau and E. M. Lifshitz, Statistical Physics, Vol. 1, Pergamon, New York (1987).

${ }^{2}$ K. Huang, Statistical Mechanics, Wiley, New York (1987).

${ }^{3}$ A. H. Carter, Classical and Statistical Thermodynamics, Prentice Hall, New Jersey (2001).

${ }^{4}$ M. H. Anderson, J. R. Ensher, M. R. Matthews, C. E. Wieman, and E. A. Cornell, Science 269, 198 (1995).

${ }^{5}$ K. B. Davis, M. O. Mewes, M. A. Joffe, M. R. Andrews, and W. Keterle, Phys. Rev. Lett. 74, 5202 (1995).

${ }^{6}$ W. Petrich, M. H. Anderson, J. R. Ensher, and E. A. Cornell, Phys. Rev. Lett. 74, 3352 (1995).

${ }^{7}$ F. Dalfovo, S. Giorgini, L. P. Pitaevskii, and S. Stringati, Rev. Mod. Phys. 71, 463 (1999).

${ }^{8}$ W. Ketterle, D. S. Dufree, and D. M. Stamper-Kurn, Making, Probing and Understanding Bose-Einstein Condensates, Preprint xxx.lanl.gov/abs/ cond-mat/9904034 (1999).

${ }^{9}$ E. A. Cornell, J. R. Ensler, and C. E. Wieman, Experiments in Dilute Atomic Bose-Einstein Condensation, Preprint xxx.lanl.gov/abs/cond-mat/ 9903109 (1999).

${ }^{10}$ N. L. Balazs and T. Bergeman, Phys. Rev. A 58, 2359 (1998).

${ }^{11}$ W. Ketterle and N. J. van Druten, Phys. Rev. A 54, 656 (1996).

${ }^{12}$ P. Navez, D. Bitouk, M. Gajda, Z. Idziaszek, and K. Rzazewski, Phys. Rev. Lett. 79, 1789 (1997).

${ }^{13}$ F. Brosens, J. T. Devreese, and L. F. Lemmens, Phys. Rev. E 55, 6795 (1997).

${ }^{14}$ Y. Castin, J. Dalibard, and C. Cohen-Tannoudji, Laser Cooling and Trapping of Neutral Atoms, in Atoms in Electromagnetic Fields, edited by C. Cohen-Tannoudji, World Scientific, Singapore (1994).

${ }^{15}$ I. S. Gradsteyn and I. M. Ryzhik, Tables of Integrals, Sums and Products, edited by A. Jeffrey, Acad. Press, New York (1994).

${ }^{16}$ E. P. Wigner, Phys. Rev. 40, 749 (1932).

${ }^{17}$ M. Fierz, Helv. Phys. Acta 29, 47 (1995).

This article was published in English in the original Russian journal. Reproduced here with stylistic changes by AIP. 